\title{
Flanking pl 0 contribution and sequence bias in matrix based epitope prediction: revisiting the assumption of independent binding pockets Christian S Parry
}

Address: Computational Biophysics Section, Laboratory of Computational Biology, National Heart, Lung and Blood Institute, National Institutes of Health, Bethesda, Maryland 20892-9314, USA

Email: Christian S Parry - csparry@helix.nih.gov

Published: 16 October 2008

BMC Structural Biology 2008, 8:44 doi:1 0.1 186/1472-6807-8-44

This article is available from: http://www.biomedcentral.com/1472-6807/8/44

(c) 2008 Parry; licensee BioMed Central Ltd.

This is an Open Access article distributed under the terms of the Creative Commons Attribution License (http://creativecommons.org/licenses/by/2.0), which permits unrestricted use, distribution, and reproduction in any medium, provided the original work is properly cited.
Received: 16 November 2007

Accepted: 16 October 2008

\begin{abstract}
Background: Eluted natural peptides from major histocompatibility molecules show patterns of conserved residues. Crystallographic structures show that the bound peptide in class II major histocompatibility complex adopts a near uniform polyproline II-like conformation. This way allelespecific favoured residues are able to anchor into pockets in the binding groove leaving other peptide side chains exposed for recognition by $T$ cells. The anchor residues form a motif. This sequence pattern can be used to screen large sequences for potential epitopes. Quantitative matrices extend the motif idea to include the contribution of non-anchor peptide residues. This report examines two new matrices that extend the binding register to incorporate the polymorphic $\mathrm{pI} 0$ pocket of human leukocyte antigen DRI. Their performance is quantified against experimental binding measurements and against the canonical nine-residue register matrix.

Results: One new matrix shows significant improvement over the base matrix; the other does not. The new matrices differ in the sequence of the peptide library.

Conclusion: One of the extended quantitative matrices showed significant improvement in prediction over the original nine residue matrix and over the other extended matrix. Proline in the sequence of the peptide library of the better performing matrix presumably stabilizes the peptide conformation through neighbour interactions. Such interactions may influence epitope prediction in this test of quantitative matrices. This calls into question the assumption of the independent contribution of individual binding pockets.
\end{abstract}

\section{Background}

It is essential to understand the host immune response in order to boost or modulate the immune system in infectious diseases, autoimmune diseases, allergies or cancer. This requires knowledge of the peptides selected and presented by class II major histocompatibility complex (MHC) molecules and the rules governing their binding and presentation to CD4+ T cells. Molecules of the MHC are surface receptors on immune cells that bind and present selected antigen as short peptides or epitopes to $\mathrm{T}$ cells with matching receptors. The peptides are produced by the proteolytic machinery of the antigen presenting cell. Class I epitopes are generated from intracellular proteins [1] and class II epitopes are processed from vesicular, endocytosed and cytosolic proteins through the exogenous pathway [2]. Processed peptides are loaded in intra- 
cellular compartments and transported to the cell surface where they are displayed for recognition by $\mathrm{T}$ cells. The unique design of the peptide binding region of the MHC and the vast polymorphism, through duplication, gene conversion and other genetic mechanisms, combine to generate hundreds of molecular variants at class I HLA-A, $-B,-C$ and class II HLA-D loci [3]. Through these mechanisms MHC molecules are able to recognize and bind a vast array of peptides with fine distinction. Each allele has a different peptide binding specificity.

The MHC receptor comprises a membrane distal peptide binding domain sitting on a scaffold or platform formed by two immunoglobulin domains juxtaposed in a characteristic fashion. The peptide binding domain consists of eight anti-parallel beta strands on top of which lie two anti-parallel alpha helices. This is a unique fold and the two alpha helices form the walls of the peptide binding cleft [4]. Polymorphic residues in the beta sheet floor and in the alpha helical walls of the MHC form pockets that enforce genetic restriction and allele specificity [5-7]. Peptides that bind to class I molecules have a restricted length, about eight to eleven residues. The bound peptide forms hydrogen bonds with conserved residues at either end of the cleft effectively sealing them. Class II molecules, on the other hand, are open at either end and allow peptides of nearly unrestricted length to extend over the termini of the binding groove.

The register of the peptide cleft or binding groove in both class I and class II MHC molecules is nine residues [8]. The positions are labelled $\mathrm{p} 1, \mathrm{p} 2, \ldots, \mathrm{p} 9$, relative to the large $\mathrm{N}$ terminal pocket in class II. Pockets p1, p4, p6 and p9 are prominent pockets in class II molecules; $\mathrm{p} 3$ and $\mathrm{p} 7$ are shallow shelves or minor pockets. Bound peptides in class II molecules adopt a polyproline type II-like conformation [9]. This near helical conformation allows the bound peptide to engage the major polymorphic pockets with anchor residues lodged in p1, p4, p6 and p9 leaving peptide side chains at p2, p3, p5, p7 and p8 simultaneously available for inspection by T cells. Peptide binding energy derives from the engagement of the peptide anchor residues in the MHC binding pockets with additional contribution from hydrogen bonds from the peptide backbone to conserved residues within the class II MHC molecule.

Methods to identify peptides that are immunogenic are important in basic and applied research - for fundamental understanding and for designing new drugs and vaccines to treat disease. Traditionally, this has meant synthesizing overlapping peptides covering the entire sequence followed by purification and direct or indirect assays of peptide binding to MHC molecules. This is time consuming and expensive. Reliable computational screening followed by experimental validation provides a more rapid and less expensive alternative. This may be carried out for several alleles to cover a wide segment of the population.

The restricting MHC molecule imposes structural constraints on the peptide anchors through polymorphic residues within the pockets [6,7]. This defines the peptide binding motif $[10,11]$ for an allele. Related alleles have overlapping peptide repertoire and share a similar motif or core sequence $[12,13]$. Anchor motifs such as obtained through eluted natural ligands and phage display libraries have been useful in epitope prediction $[12,14,15]$. Quantitative matrices are extended motifs. They are an improvement over simple motifs and provide more informational content. Their coefficients describe the likelihood of an amino acid at a given location in a peptide to contribute to binding to an MHC allele [16-18]. They give high specificity, are fast, intuitive and easy to use.

Quantitative matrices are typically $20 \times 9$ in dimension reflecting the twenty natural amino acids and the nine residue register of the binding cleft of MHC molecules [1618] as has been determined by X-ray crystallography [8]. The overall binding energy of the peptide is assumed to be the linear sum of the contribution of individual side chains. The peptide backbone hydrogen bonding contribution is ignored. This scheme is the independent binding of side chains approximation [16]. Equivalent matrices have been derived from the relative abundance of an amino acid at a given position from the sequence alignment profile of a library of known binders to an MHC allele [19]. Epitope prediction by quantitative matrices has been useful in identifying antigens in allergens [20], infectious agents such as Mycobacterium tuberculosis [21], and tumours [22]. Reviews by Korber and colleagues and by Tsurui and Takahashi give a current survey of the field $[23,24]$.

Most prediction programs, whether they use quantitative matrices or machine learning methods, employ the canonical nine pocket binding register. There is growing appreciation that flanking residues influence peptide binding [25]. Flanking residues at the C-terminus of the binding groove of class II MHC molecules are polymorphic. The shelf formed at flanking position p10 makes potential sequence dependent contribution toward peptide binding energy. The equivalent flanking position at the $\mathrm{N}$-terminus of the peptide groove is conserved; therefore the contribution of the position preceding pocket $\mathrm{p} 1$ (p-1) is ignored.

Results from structural and binding studies using the peptide AWCSDEALPLGSPRCD in complex with HLADRB3*0101 (HLA-DR52a) show a contribution from position p10. 24-AWCSDEALPLGSPRCD-39, from the N- 
terminus of human integrin $\alpha 2 \beta I I I a$ is a major ligand of DR52a. A natural variant of the epitope widely distributed in the population is 24-AWCSDEALPPGSPRCD-39. The Leu33/Pro33 dimorphism is the basis of the unidirectional alloimmune posttransfusion thrombocytopenia purpura (PTP) and fetal-maternal alloimmune thrombocytopenia (FMAIT), severe blood disorders in some DR52a subjects homozygous in Pro33 [26-28]. While AWCSDEALPPGS, or the second epitope 24-AWCSDEALPPGSPRCD-39 (Pro33), does not bind to DR52a AWCSDEALPPLS (leucine replacing glycine at position 10) has been demonstrated to bind. This has been confirmed by crystallographic studies (CSP, unpublished). These point to the contribution of leucine as an auxiliary anchor and the importance of position $\mathrm{p} 10$. Other studies of peptide-class II MHC complexes show a sequence dependent role for p10 [29].

In this study, we examine whether adding the contribution of position $\mathrm{p} 10$ improves prediction accuracy over a common 9-pocket matrix [17]. We construct two new quantitative scoring matrices for HLA-DR1 extended to include the contribution of flanking position p10. Coefficients for position 10 were constructed from the peptide library AAYSDQATPLLXSPR, where $\mathrm{X}$ at position p10 is one of the twenty natural amino acids. Canonical anchor residues are in bold. The base peptide was derived from the N-terminal human integrin $\alpha 2 \beta I I I a$ peptide used for DR52a studies and has been designed to bind in a specific frame to HLA-DR1 by substituting well known anchor residues from the DR1 peptide binding motif and crystallographic studies [30,31]. A second peptide library was based on AAYSDQATLLLXSPR where a proline has been replaced with leucine in order to avoid conformational effects due to proline. We find that the new matrix based on AAYSDQATPLLXSPR (called PP10, from the role of position 10 and the extra proline) shows significant improvement over the original nine residue register matrix, P9. The matrix based on AAYSDQATLLLXSPR (P10, from the role of position 10) does not show such improvement. The peptide libraries for the extended matrices differ in sequence at one position: proline versus leucine.

\section{Results \\ Bioinformatic analysis}

We have used $\mathrm{IC}_{50}$ values, obtained from binding assays of a variety of test peptides to DR1, to construct coefficients to extend the Hammer 9-register matrix [17] (P9) to position 10 of the peptide binding cleft. The protocol is described under Methods. The new extended matrices, $P 10$ and PP10, are validated in regression analysis of predicted values against experimental binding measurements of peptides from glutamic acid decarboxylase (GAD65), islet cell antigens (ICA69) [32] and Varicella-zoster virus
(VZV) (unpublished data). We compare these with results using the canonical 9-register matrix for DR1 [17]. The TEPITOPE virtual matrices for DR1 and other alleles [33] are based on this published matrix according to similarity in the pocket sequence profile. Logarithm of $\mathrm{IC}_{50}$ values are plotted against predicted values and a function is fit to the data. A fitting function is chosen whose coefficients minimize the residuals.

The best fit was found to be a straight line. Higher order polynomial functions were tried but gave worse results. Low $\mathrm{IC}_{50}$ values correspond to good binding and high $\mathrm{IC}_{50}$ values correspond to poor binding, and negative predicted values. A flat fit has no predictive use. Plots for GAD65, ICA69 and VZV are shown in Figures 1 and 2. In all three scoring methods, there are both false positives (upper right quadrant) and false negatives (bottom left quadrant, Figures 1 and 2). False positives can be screened out in validation tests but false negatives are problematic. With these data sets, the scoring matrices show few false negatives. This is a useful property and the matrices can be used to screen large sequences without missing potential epitopes. A high threshold may be set to eliminate falsely predicted peptides.

In all three data sets, GAD65, ICA69 and VZV, for the three matrices tested there is correlation between binding (measured $\mathrm{IC}_{50}$ ) and predicted values (Figure 1). This indicates predictive value in all three matrices, the two new extended basis matrices P10 and PP10 and the control matrix P9. For GAD65 (Figure 1a), matrices of the original nine-residue matrix $(P 9)$ and the extended matrices (P10 and PP10) perform about equally from the fits. Using the slope $m$ as a surrogate measure, PP10 is better than $P 9$ ( 0.383 vs. 0.352 , in absolute values) and $P 9$ is in turn better than matrix $P 10$ ( 0.352 vs. 0.341$)$. The analysis is summarized in Table 1. For ICA69 (Figure 1b), PP10 and $P 9$ have nearly the same slope $(0.327$ vs. 0.338$)$ and better predictive power than $P 10(0.275)$. The difference between the respective slopes of $P 9$ and $P P 10$ is not significant but the deviation between 0.327 and 0.338 (PP10 and $P 9$ ) and 0.275 for $P 10$ appears significant. Using the coarse criterion of slope $m$ for VZV (Figure 1c), $P 9$ is better than P10 (0.252 vs. 0.225). Visual inspection of the graphs supports these assertions (Figure 1). Interestingly, from Table 1, the difference in the absolute slope values between $P 9$ and P10 for GAD65 and ICA69 data sets is 0.011 . Difference in peptide-HLA-DR binding assay sensitivity between different antigenic proteins, data sets, individual experiments and the nature of competition assays makes it difficult to establish absolute numbers, and comparison between different data sets is difficult. Comparison within a data set is more valid as revealed by the plots. 
A

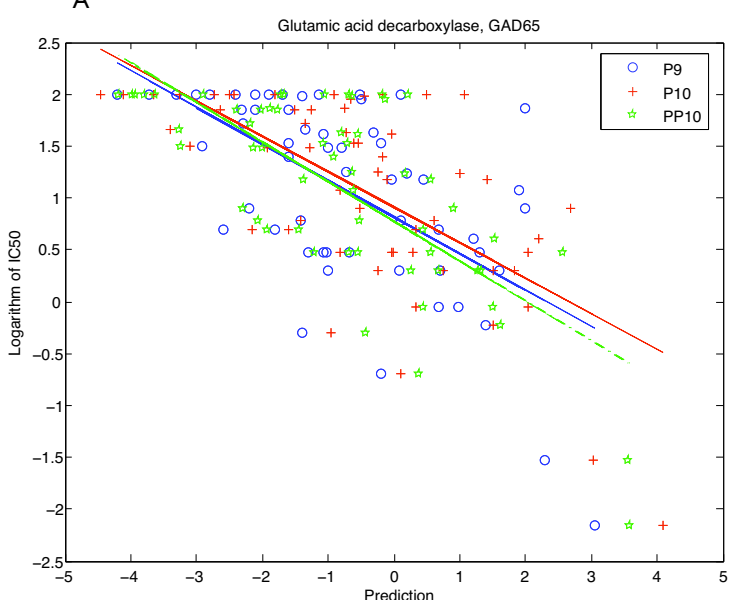

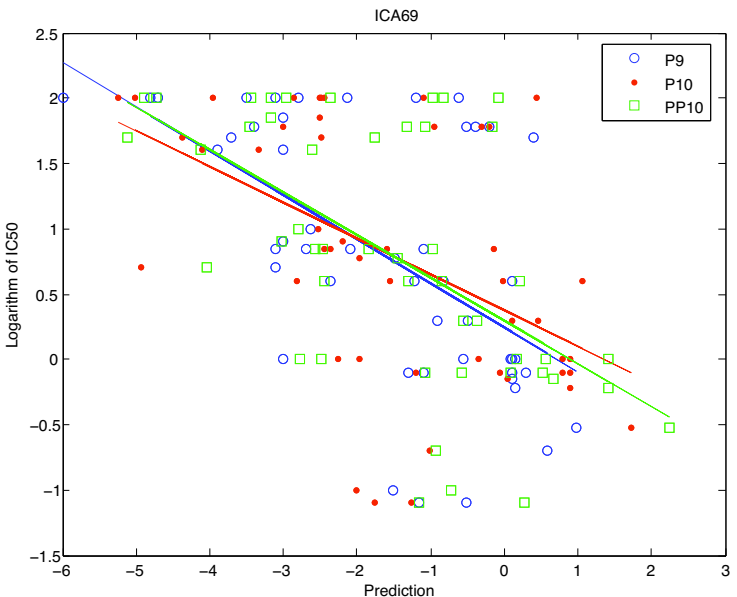

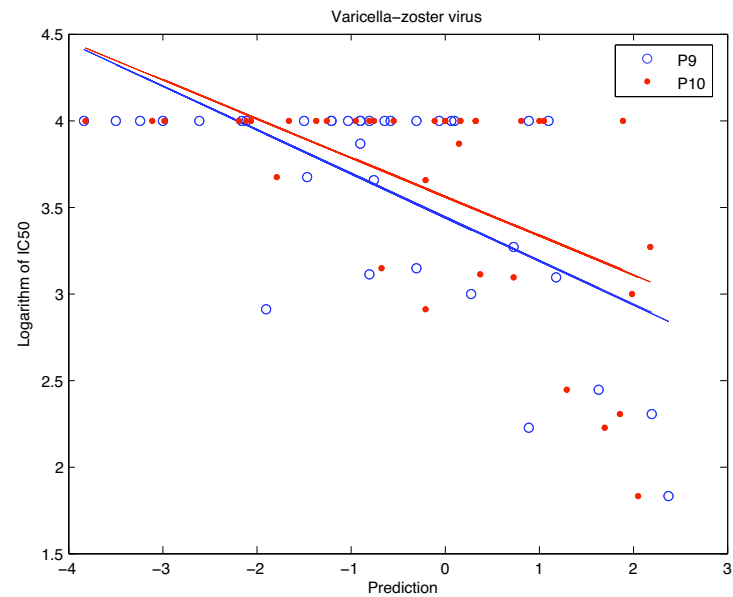

\section{Figure I}

Plots of binding measurements versus prediction values. Half maximal inhibitory concentration (IC50) values of peptide sequences are plotted as a function of their predicted values for each of the three matrices, P9 (open circles, blue), PIO (cross, red) and PPIO (stars, green). A line is fitted to the plotted values in the respective colors. This is done for data sets A. Glutamic acid decarboxylase, GAD65; B. Islet cell antigen protein, ICA69; and C. Varicella zoster virus, VZV.
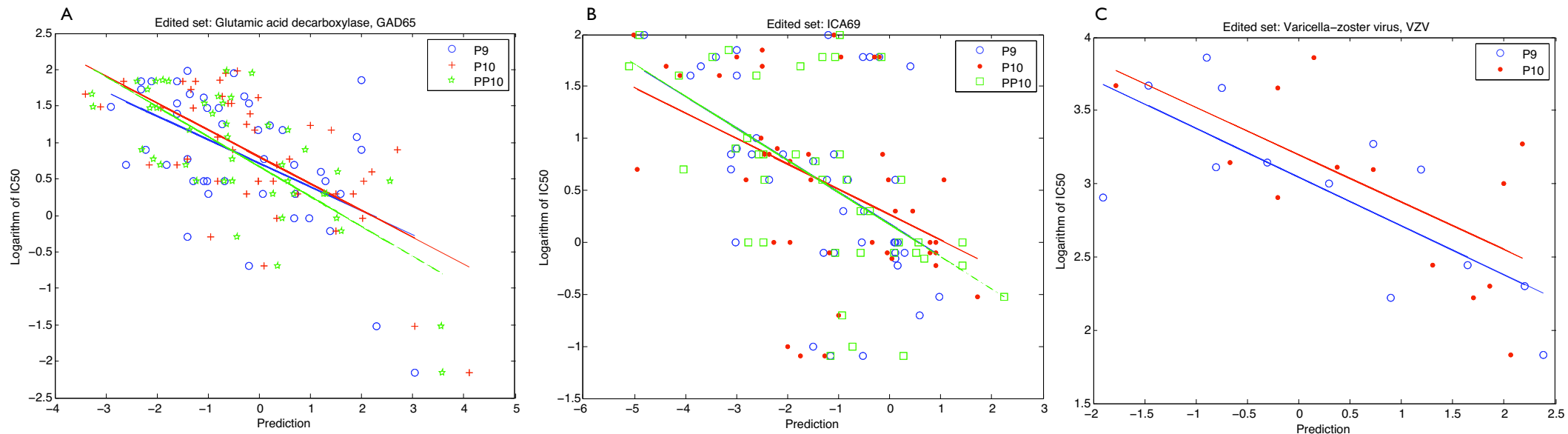

Figure 2

Plots of edited binding measurements versus prediction values. Binding measurements are again plotted against predicted values. Poor binders have been removed, and half maximal inhibitory concentration (IC50) values of peptide sequences are plotted for each of the three matrices, P9 (open circles, blue), PIO (cross, red) and PPIO (stars, green). A line is fitted to the plotted values in the respective colors for the respective data sets, A. Glutamic acid decarbolase, GAD65; B. Islet cell antigen, ICA69, and C. Varicella zoster virus, VZV. 
Table I: Summary of analysis

\begin{tabular}{lllll}
\hline A. Full data & & & & \\
\hline Data set & method & $-m$ & $J$ & $r^{2}$ \\
\hline \multirow{2}{*}{ GAD65 } & P9 & 0.352 & 30.22 & 0.373 \\
& PIO & 0.341 & 26.75 & 0.445 \\
& PPIO & 0.383 & 22.37 & 0.536 \\
ICA69 & $P 9$ & 0.338 & 29.91 & 0.325 \\
& $P 10$ & 0.275 & 33.24 & 0.250 \\
& $P P 10$ & 0.327 & 29.27 & 0.340 \\
VZV & $P 9$ & 0.252 & 8.16 & 0.386 \\
& $P 10$ & 0.225 & 8.99 & 0.324 \\
\hline
\end{tabular}

B. Data edited to remove poor binders

\begin{tabular}{llccc}
\hline Data set & method & $-m$ & $J$ & $r^{2}$ \\
\hline \multirow{2}{*}{ GAD65 } & P9 & 0.324 & 25.44 & 0.279 \\
& PIO & 0.368 & 20.12 & 0.429 \\
& PPIO & 0.410 & 16.12 & 0.543 \\
ICA69 & P9 & 0.308 & 24.27 & 0.251 \\
& PIO & 0.244 & 25.47 & 0.214 \\
& PPIO & 0.308 & 21.22 & 0.345 \\
VZV & P9 & 0.334 & 1.92 & 0.573 \\
& PIO & 0.324 & 2.56 & 0.431 \\
\hline
\end{tabular}

The fit of prediction to binding has been calculated for each prediction method using measures of slope $(-m)$, sum of the square terms of the residual error $(V)$ and $r$-square value $\left(r^{2}\right)$ for peptide sequences from GAD65, ICA69 and VZV. Table IA shows the statistics for the unedited data, and Table IB shows the equivalent statistics for the data edited to remove poor binding measurements.

ures $1 \mathrm{a}, \mathrm{b}$ and $1 \mathrm{c}$ show that low binding peptides $\left(\mathrm{IC}_{50}=\right.$ $100 \mathrm{uM}$ for GAD65 and ICA69 and $10 \mathrm{uM}$ for VZV) are poorly predicted. This marks the limit of sensitivity of the experimental measurement. The analysis is repeated without these points: Figures $2 \mathrm{a}, \mathrm{b}$ and $2 \mathrm{c}$ show the same plots when data points representing poor $\mathrm{IC}_{50}$ values are ignored. It is not clear how significant the difference between $P 9$ and P10 slopes is for GAD65. Nevertheless, the conclusion that PP10 is superior is not changed. The VZV data set further reveals the difference in performance between P9 and P10. The magnitude of the slope used this way calibrates closely the "structure" or "informational potential" within the data; a flat fit signifies random data and has neither "structure" nor predictive potential.

The predictive potential is more accurately captured in terms of variance ("bandwidth") and residuals ("noise"). Residuals for the three data sets were calculated. A typical plot is shown in Figure 3 for GAD65 data set. The sum of the square of the residual values is calculated for each prediction matrix P9, P10 or PP10. Finally, the quality of the fit is assessed through the $r$-square value:

$$
r^{2}=\left(1-\frac{J}{S}\right)
$$

$J$ is the sum of the square terms of the residual error and $S$ is the variance of the data. The analyses incorporating the slope $m$ of the fitting function, residuals $J$, and $r^{2}$ are shown in Table 1. $r^{2}$ values range from 0.25 to 0.54 for the full data and 0.21 to 0.57 for the pruned set. The corresponding range for the magnitude of the slope is 0.22 to 0.38 for the full set and 0.24 to 0.41 for the edited set. The slopes shadow closely the respective $r^{2}$ values (Table 1 ).

From $r^{2}$ values, prediction method PP10 again works the best in both GAD65 and ICA69 data sets: PP10 performs better than the control matrix $P 9$ and is superior to the other extended basis matrix P10. P10 is better than the control $P 9$ matrix in GAD65 ( $r^{2}$ of 0.45 versus 0.37$)$ but not in ICA69 ( 0.25 versus 0.33$)$. The ordering and conclusions are unchanged with filtered data. PP10 matrix has superior discrimination potential in terms of the slope $m$ and also in terms of $r^{2}$. PP10 shows marked improvement over matrix P9 and PP10 is also better than P10. While extended matrix $P 10$ makes better prediction than $P 9$ in GAD 65 it is worse than P9 in the edited ICA69 data set. In VZV, matrix $P 10$ shows no improvement over $P 9$ in either the filtered or unfiltered data sets, according to $r^{2}$. Overall, average $r^{2}$ values of the raw data sets (Table 1) are 0.36, 0.34 and 0.44 respectively for $P 9, P 10$ and $P P 10$. For comparison, reported average $r^{2}$ value of a large-scale evaluation of class II molecules using similar quantitative matrices is $0.25[34]$.

\section{Structural analysis}

To gain structural insight into epitope prediction, and why matrix PP10 performs better than P9, we examined peptides that bind well according to $\mathrm{IC}_{50}$ binding measurements. The best experimental binding measurement is for peptide 54 from GAD65 (GDKVNFFRMVISNPAATHQD, $p \mathrm{IC}_{50}=2.15$; Supplementary table, Additional file 1). The same register FRMVISNPA(A) is predicted as a strong binder for both matrices, P9 (predicted score = 3.05 ) and PP10 (predicted score $=3.58$ ). Anchor residues at dominant pockets p1, p4, p6 and p9 are as expected for DR1 [12]. Ala at position p10 is also predicted to contribute to favourable binding. Incidentally, matrix P10 is in agreement: the same binding frame is predicted and Ala at the extended position is also predicted to interact with the shallow shelf at position p10 for enhanced binding. The added contribution of Ala is given greater weight in matrix P10 (1.05 units) than in PP10 (0.53 units). Competition binding assay of the peptides used for the libraries shows that Ala is a preferred residue at p10 for HLA-DR1. 

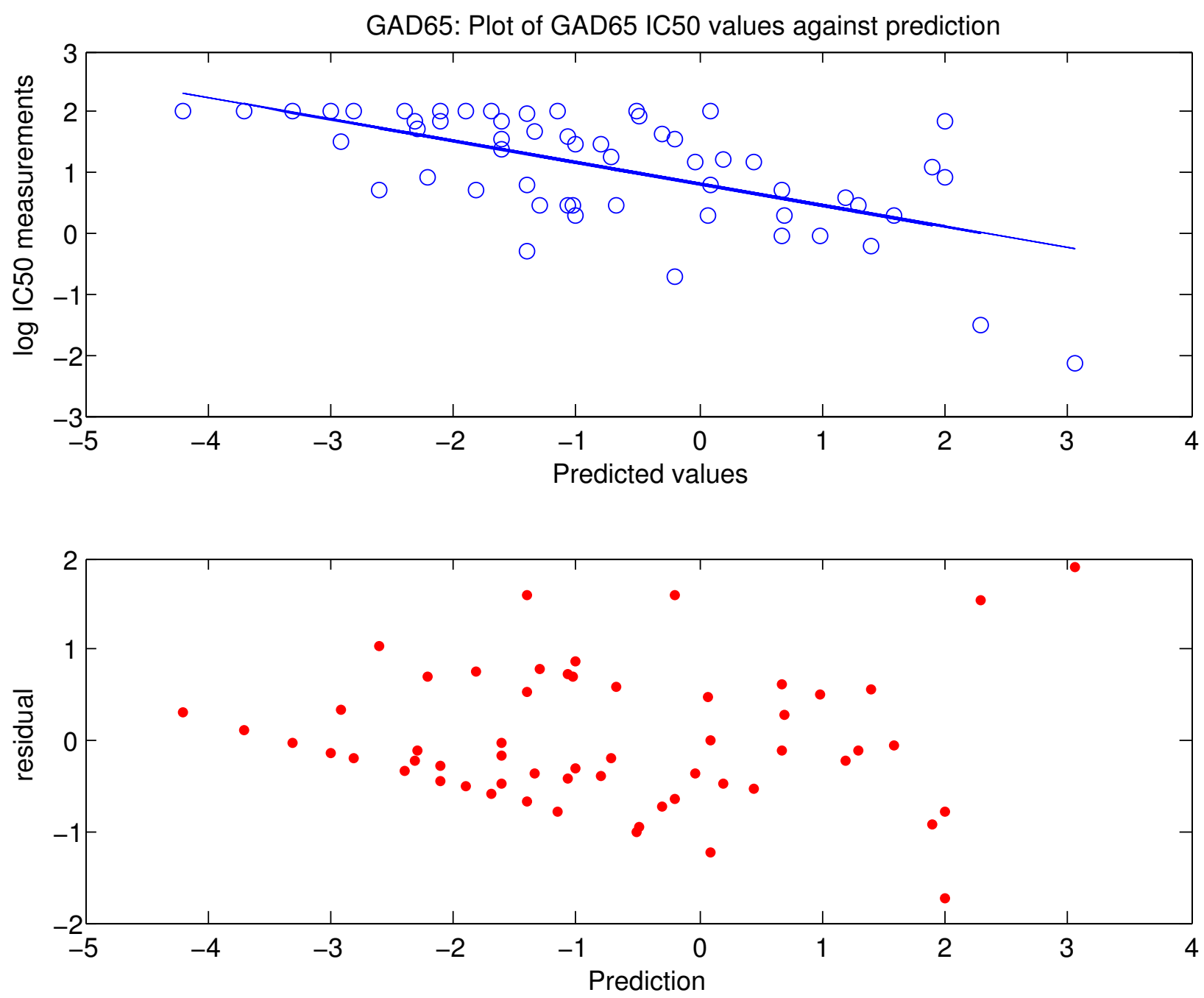

Figure 3

Distribution of residuals for GAD65. Values of half maximal inhibitory concentration are plotted for glutamic acid decarboxylase (GAD65) together with calculated residuals. The top panel is the plot of IC50 against prediction for GAD65; the bottom panel shows the distribution of the residuals.

A unanimous binding frame (in bold letters) is also predicted from peptide 9 (Supplementary table, Additional file 1) SCSKVDVNYAFLHATDLLPA, another strong binder. Leucine at position p10 is predicted to contribute to greater binding affinity, in agreement with experiment. Matrices $P 9$ and $P P 10$ predict the same frame (in bold letters) in peptide 4, QVAQKFTGGIGNKLCALLYG (Supplementary table, Additional file 1). Strong peptide anchor residues at $\mathrm{p} 1$ and $\mathrm{p} 9$ ( $\mathrm{F}$ and $\mathrm{L}$, respectively) compensate for poor anchors $(\mathrm{G})$ at $\mathrm{p} 4$ and $\mathrm{p} 6$, and the contribution of cysteine at position p10 is predicted to enhance bind- ing from matrix $P P 10$. The predicted binding frame in matrix P10, however, differs - IGNKLCALLY.

Another example shows how the extended position changes the predicted binding frame between matrices $P 9$ and PP10: in SHFSLKKGAAALGIGTDSVI (peptide 28), LKKGAAALG is predicted to bind best by matrix $P 9$ while FSLKKGAAAL is predicted by the two extended basis matrices. LKKGAAALG (predicted by P9) has a good leucine anchor at $\mathrm{p} 1$ but three poor anchors at $\mathrm{p} 4, \mathrm{p} 6$ and $\mathrm{p} 9$. For matrices $P 10$ and $P P 10$, a strong phenylalanine anchor 
at position $\mathrm{p} 1$ in the peptide predicted combines with lysine at $\mathrm{p} 4$ and the auxiliary leucine anchor at position 10 to improve the score.

The peptide VETFRHRAISDTWLTVNRME from ICA69 is another example where the extended matrix PP10 predicts a frame (FRHRAISDTW, score $=0.27$ ) different from that predicted by P9 (VETFRHRAI, score $=-1.15$ ). The better score predicted by PP10 may arise from an aromatic hydrophobic anchor in pocket 1 versus a relatively small aliphatic $\mathrm{p} 1$ anchor, valine, predicted by matrix $P 9$. In the latter, phenylalanine is disfavoured in pocket 4 , and likewise histidine in pocket 6 , in the frame predicted by matrix $P 9$. The other extended matrix $P 10$ predicts the same frame as PP10 but with a much worse score $(-1.75)$. This implies a predicted negative contribution of tryptophan at position p10 for matrix $P 10$ but a positive contribution of tryptophan for PP10. Experimental binding measurements support a positive contribution of tryptophan at pocket 10 . The binding frame predicted by matrix PP10 is structurally consistent with the measured binding affinity.

In peptide 117, the ICA69 peptide QCRTEYRGALLWMKDVSQEL (Supplementary table, Additional file 1) is predicted to bind in a different frame (CRTEYRGAL, score = 0.52 ) than that predicted for PP10 (YRGALLWMKD, score $=-1.15$ ). Non-ideal anchors (Cys at p1 and Glu at p4) predicted by matrix $P 9$ are compensated by good anchors (Arg at p6 and Leu at p9). The frame predicted by PP10 possesses a large and favourable hydrophobic anchor (Tyr) at p1, a favoured small residue (Ala) at p4 but Leu and Lys at pockets p6 and p9, respectively. Leu and Lys are disfavoured in these positions. The contribution at position p10 does not overcome these hindrances.

AIHESFKGYQPYEFTTLKSL and RKESSSFKTEDGKSILSALD (peptides 126 and 132; Supplementary table, Additional file 1) are predicted as strong binders, in agreement with experimental data, and in the expected registers, FKGYQPYEF(T) and FKTEDGKSI(L), for all three matrices. In peptide 126, polar threonine is not favoured at position $\mathrm{p} 10$; this reduces the score slightly in both extended matrices. On the other hand, in peptide 132, hydrophobic leucine anchor at p10 in the predicted fragment enhances the interaction between DR1 and the peptide ligand to give a slightly higher score for both extended matrices $P 10$ and PP10.

And in another example, the predicted register is shifted one position between matrices $P 9$ and PP10 for peptide SVVNKMQQRYWETKQAFIKA (peptide 103; Supplementary table, Additional file 1): VNKMQQRYW and VVNKMQQRYW (p1, p4, p6 and p9 anchors are in bold). The added basis in PP10, the increased number of parameters, has allowed better prediction accuracy.

Finally, all three matrices unanimously select the same best frame YGAFDPLLA(V) from overlapping peptides 33 and 34 (Supplementary table, Additional file 1). The experimental pIC50 values are 0.05 (peptide 33) and 0.70 (peptide 34) compared to predicted scores of 0.68 , 0.33 and 0.45 for P9, P10 and PP10, respectively. Similarly, the same frame FRKVQTQVRL is unanimously selected by the three matrices for peptides 119 and 120 (Supplementary table, Additional file 1). pIC50 values are 0.22 (peptide 119) and 0.00 (peptide 120). Predicted scores are $0.15,0.90$ and 1.42 for P9, P10 and PP10, respectively, for the unanimously predicted best frame for the two peptides. In the former pair of peptides, the experimental values are far apart while the computational scores are relatively close. In the latter set, the experimental values are close but the predicted scores show a big spread.

Statistics for predicted scores are easy to obtain but similar statistics for binding measurements are difficult to obtain as little statistical data is given along with the experimental data. As is typical of such measurements, baseline values are determined on a case by case basis.

\section{Discussion}

An important contribution to the binding affinity of peptide to class II MHC is the aggregate of the interaction of individual peptide side chains with the polymorphic pockets. The contribution of backbone atoms is ignored as is formalized in the independent binding of pockets hypothesis [16] that underlies epitope prediction algorithms based on quantitative matrices. Methods of epitope prediction, including quantitative matrices, typically consider only the nine positions or pockets within the binding groove. There are reports that show, however, that sequences flanking the binding cleft affect peptide binding affinity [25]. This contribution when accounted for may therefore improve epitope prediction accuracy for class II MHC alleles. The termini of the binding groove of class I molecules are sealed.

We constructed two matrices P10 and PP10 that incorporate flanking position $\mathrm{p} 10$ and tested them against experimental binding data. Extended matrix PP10 showed significant improvement over the canonical 9-mer matrix, $P 9$. In validation tests of two typical data sets extended matrix PP10 showed superior performance (average $r^{2}=$ 0.440 ) over the original P9 matrix (average $r^{2}=0.350$ ). On the same test sets, extended matrix $P 10$ performed no better (average $\left.r^{2}=0.350\right)$ than $P 9$ (Table 1$)$. 
The superior performance of prediction matrix PP10 is best explained in terms of additional position $\mathrm{p} 10$ in the binding register. This explicitly increases the number of parameters. A major conclusion therefore is that incorporating the flanking position may improve class II MHC epitope prediction. Extended matrix PP10 also selects a more plausible binding register than what is predicted by matrix $P 9$. The relative poor performance of matrix $P 10$ is best explained in terms of the difference in sequence of the peptide library it was based on. It is not clear the effect of the -LLL- subsequence (in P10) on the peptide conformation. Conversely, it is conceivable that the better performance of matrix PP10 derives from greater stabilization of the peptide polyproline II-like conformation by the extra proline in the sequence of the peptide library.

The left handed polyproline II helical conformation is found in structural proteins, in signalling molecules and as ligands in MHC complexes [9]. Proline is unusual among natural amino acids. In a polypeptide, its $\phi$ torsion angle is restricted to $-63^{\circ}$. The $\delta$ carbon of the proline ring interacts with the backbone $\mathrm{N}$ and constrains the preceding residue [35]. Such influence of proline on the adjacent residue is contrary to the assumption of independent binding of peptide side chains and needs accounting in quantitative scoring matrices.

Many lines of structural studies of peptide-MHC complexes suggest cooperative interactions between pockets [36]. Peptide selection and binding may be compared to sampling the conformational space available to the complex of peptide and MHC. The path followed and the final structure adopted by the complex depend on sequence, and these involve cooperative interactions. The accompanying conformational changes are not accounted for in quantitative matrices. A related issue is the stability of the bound complex.

Hydrogen bonds from the peptide backbone are a major contribution to peptide-class II MHC binding. Experimental structures show 12-16 such bonds from the peptide backbone to usually conserved residues in the class II molecule. Each such hydrogen bond is important for binding affinity and stability [37]. While, in principle, these backbone hydrogen bonds enable peptide binding regardless of sequence the number of such bonds may vary depending on the peptide sequence. Proline residues in a peptide sequence can alter the pattern and number of such backbone hydrogen bonds. Neither quantitative matrices nor the assumption of independent binding takes backbone hydrogen bonding into account.

Further, a given peptide-class II complex is best represented as a heterogeneous set of conformations of varying stability. These conformational dynamics are a function of the particular peptide in the MHC and reflect sequence, near neighbour and long range pocket interactions. This is another limiting factor in epitope prediction. Already, sequence dependence is assumed in functional work and sequence sensitivity to selectivity and stability of class II ligands has been demonstrated in the catalysis of peptide loading down to the level of hydrogen bonds $[36,38,39]$.

Peters and colleagues have proposed the method of stabilized matrices [40] to account for near neighbour and cooperative interactions between pockets. Their calculated coefficients are used to augment the well known quantitative matrices used in TEPITOPE [33]. The added terms are small, about a factor of 10 smaller than the entries of the original quantitative matrices. However, stabilized matrices improved prediction performance over the original quantitative matrix, and to a level equalling or exceeding that of general (machine learning) methods.

This report does not address questions about peptides that are poorly predicted. Experimental limitations are a factor in poor predictions but are difficult to quantify. Measured $\mathrm{IC}_{50}$ values are relative to a reference peptide, and sensitivity may vary from assay to assay. It is also not always clear what is binding. If bound peptide is what is being measured, other questions arise such as in what frame. An examination of crystallographic structures of peptide-class II MHC complexes shows that peptide anchors are not always fully engaged in binding pockets especially after pocket $\mathrm{p} 6$; in the collagen peptide complex with DRB5*0101 [41] the peptide side chain at p9 is out of pocket and extends rather to the shelf at position 10 . In general, not all pockets need to be fully engaged for good peptide binding [42]. Short of experimentally derived structures exactly how peptides bind in each complex, or whether they bind at all in the binding site, as measured in assays, has to be assumed. These issues are intimately linked with the biology of the system.

The biology and the experimental restriction of the system are important limiting factors in $\mathrm{T}$ cell epitope prediction accuracy. Immunoassays need to become reliably quantitative in order to ensure accuracy in prediction. Heterogeneity of peptide conformations also needs to be addressed. These are difficult issues but they must be taken into account in interpreting results from epitope prediction.

\section{Conclusion}

This study set out to find whether the contribution of flanking pocket 10 improves prediction accuracy. Two matrices incorporating position p10 were constructed. Tests showed that the contribution of the added basis describing position p10 may lead to improved prediction 
over the usual nine residue register matrix. The added dimension explicitly increases the parameters. The study also revealed the importance of sequence context.

The aim of modern biology is to explain physiological phenomena not only at the level of cells and tissues but also at the molecular level. This requires full knowledge of the coding sequence and the atomic structure of the molecules. Nevertheless, the three dimensional representation of molecules of itself is inadequate. Near neighbour interactions between residues, long range cooperative effects between domains and other tempo-spatial effects are just as important, hence a need for representations that capture these.

Quantitative matrices used in epitope prediction are actually an example of such parameterization. A matrix coefficient contains parameterization of the physical chemical properties of pocket shape, peptide side chain to pocket interactions and electrostatics without explicit description. Sequence dependence is considered on the basis of individual pockets. The approach followed here similarly aims at increasing the parameters of the scoring matrix by explicitly increasing the basis through flanking position p10. The method of stabilized matrices has demonstrated a way of introducing small additional terms describing cooperative effects [40].

Comparing the performance of the two extended matrices described here has made evident implicit sequence bias in the matrices. Proper accounting of sequence effect will further contribute to prediction accuracy. Peptide libraries are implicitly optimized through the incorporation of favourable residues at anchor positions; these capture salient nonlinear features of peptide conformations. Fuller understanding may lead to a judicious choice of basis, parameters or peptide library to construct scoring matrices that capture more of these cooperative interactions to improve prediction accuracy.

\section{Methods \\ Generating coefficients}

Matrix coefficients to extend the binding register to position p10 are derived following the method described previously [17]. A designed peptide carrying the test amino acid residue at position p10 is used in competitive human leukocyte antigen binding assays with purified DR1 allele. The peptide library was designed after the human integrin $\beta 3$ peptide AWCSDEALPLGSPRC, a natural ligand of HLA-DR52a (DRA/DRB3*0101), and altered to incorporate anchor residues favourable to HLA-DR1, Y, Q, T and $\mathrm{L}$, as deduced from the known peptide binding motif [30]. The first series called PP10 is AAYSDQATPLLXSPR; anchor residues, at positions 1, 4, 6 and $9(\mathrm{p} 1, \mathrm{p} 4, \mathrm{p} 6$ and $\mathrm{p} 9)$ in bold, and $\mathrm{X}$ represents one of the twenty natural $\mathrm{L}$ amino acids. In order to obtain coefficients for a residue $\mathrm{X}$ at $\mathrm{p} 10$, the amino acid at that position within the peptide is varied and the $\mathrm{IC}_{50}$ value is measured (described below). The $\mathrm{IC}_{50}$ value is normalized with the $\mathrm{IC}_{50}$ value of the Alasubstitution at position $\mathrm{p} 10$ for each residue $\mathrm{X}$. The logarithm of the reciprocal value of normalization is obtained as:

$$
\log \left(\frac{\mathrm{IC} 50_{A l a}}{\mathrm{IC} 50_{X}}\right)
$$

Another peptide library is constructed based on the peptide AAYSDQATLLLXSPR, P10. Proline has been replaced with leucine to avoid the backbone restriction and conformational heterogeneity of the pyrrolidine ring that are associated with proline. The extended matrix coefficients are calculated independently for pocket 10 with no assumption of neighbouring pockets or peptide positions. The free energy of binding of a peptide is approximated by the sum of individual side chain contribution within the binding register, the Independent Binding of Side Chains [16].

\section{Peptide binding experiments}

The affinity of test peptides of the peptide library to HLADR1 was determined in standard competition binding experiments. $25 \mathrm{nM}$ of the unliganded class II molecule was incubated with an equimolar amount of the probe, biotinylated influenza virus HA [306-318] peptide and unlabelled competitor peptide; the amount of the competitor peptide used is varied for each assay. The assay mixture is incubated in $50 \mathrm{mM} \mathrm{NaCl}, 100 \mathrm{mM} \mathrm{Na}_{3} \mathrm{PO}_{4}$, $\mathrm{pH}=5.5$, a cocktail giving final concentration $1 \mathrm{mg} / \mathrm{ml}$ PMSF, $37 \mathrm{ug} / \mathrm{ml}$ iodoacetamide, $10 \mathrm{mM}$ EDTA, 0.02\% $\mathrm{NaN}_{3}$ and $0.5 \mathrm{mg} / \mathrm{ml}$ octylglucoside at $37^{\circ} \mathrm{C}$ until equilibrium is reached after 3 days. This was followed by immunoassay using the anti-HLA-DR1 antibody LB3.1 in streptavidin to detect the bound biotinylated HA [306318] peptide. $\mathrm{IC}_{50}$ values are deduced for each peptide from fluorescence values with respect to a range of concentration of the competitor peptide [31].

\section{Test set}

The performance of the extended matrices was evaluated in prediction against experimental binding measurements of peptides derived from $65 \mathrm{kDa}$ glutamic acid decarboxylase (GAD65) [32], $69 \mathrm{kDa}$ islet cell antigens (ICA69) [32] and Varicella-zoster virus (VZV) (unpublished data). GAD65 and ICA69 data consist of 20-mer peptides overlapping by 10 amino acids and covering the complete sequence of the proteins. VZV peptides contain a variety of 15-mer through 27-mer peptides also overlapping by 10 amino acids and covering the entire range of the sequence. 


\section{Validation}

For each data set, a dependent variable was derived from experimental $\mathrm{IC}_{50}$ values through a logarithmic transform. This is correlated with predicted peptide scores through a fitted function by regression. Linear and nonlinear functions are used and evaluated based on the residual. The predicted scores are further evaluated through the $r^{2}$ (Rsquare) function. Regression analysis was carried out using MATLAB http://www.mathworks.com. Predictions were carried out within the in-house MHC epitope prediction tool PREDICT (CSP, unpublished).

\section{Authors' contributions}

CSP wrote the prediction platform, tested and installed it. He programmed further for the extended matrices project; carried out the analysis; wrote and edited the manuscript.

\section{Additional material}

\section{Additional File 1}

Supplementary table

Click here for file

[http://www.biomedcentral.com/content/supplementary/14726807-8-44-S1.doc]

\section{Acknowledgements}

This research was supported in part by the Intramural Research Program of the National Heart, Lung and Blood Institute, National Institutes of Health. I am grateful to Dr. Bernard R. Brooks for his help and support.

\section{References}

I. Kloetzel PM: Antigen processing by the proteasome. Nat Rev Mol Cell Biol 200I, 2: 179-I87.

2. Watts C: The exogenous pathway for antigen presentation on major histocompatibility complex class II and CDI molecules. Nat Immunol 2004, 5:685-692.

3. Trowsdale J: Genomic structure and function in the MHC. Trends Genet 1993, 9:1 17-122.

4. Bjorkman PJ, Saper MA, Samraoui B, Bennett WS, Strominger JL, Wiley DC: Structure of the human class I histocompatibility antigen, HLA-A2. Nature 1987, 329:506-512.

5. Bjorkman PJ, Saper MA, Samraoui B, Bennett WS, Strominger JL, Wiley DC: The foreign antigen binding site and $T$ cell recognition regions of class I histocompatibility antigens. Nature | 987, 329:5 | 2-518.

6. Garrett TP, Saper MA, Bjorkman PJ, Strominger JL, Wiley DC: Specificity pockets for the side chains of peptide antigens in HLA-Aw68. Nature 1989, 342:692-696.

7. Zinkernagel RM, Doherty PC: Restriction of in vitro T cell-mediated cytotoxicity in lymphocytic choriomeningitis within a syngeneic or semiallogeneic system. Nature 1974, 248:701-702.

8. Madden DR: The three-dimensional structure of peptideMHC complexes. Annu Rev Immunol 1995, 13:587-622.

9. Jardetzky TS, Brown JH, Gorga JC, Stern LJ, Urban RG, Strominger JL, Wiley DC: Crystallographic analysis of endogenous peptides associated with HLA-DRI suggests a common, polyproline II-like conformation for bound peptides. Proc Natl Acad Sci USA 1996, 93:734-738.

10. Buus S, Sette A, Colon SM, Miles C, Grey HM: The relation between major histocompatibility complex (MHC) restriction and the capacity of la to bind immunogenic peptides. Science 1987, 235:1353-1358.
II. Sette A, Buus S, Colon S, Smith JA, Miles C, Grey HM: Structural characteristics of an antigen required for its interaction with la and recognition by T cells. Nature 1987, 328:395-399.

12. Hammer J, Takacs B, Sinigaglia F: Identification of a motif for HLA-DR I binding peptides using MI3 display libraries. J Exp Med 1992, 176:1007-1013.

13. Malcherek G, Gnau V, Jung G, Rammensee HG, Melms A: Supermotifs enable natural invariant chain-derived peptides to interact with many major histocompatibility complex-class II molecules. J Exp Med 1995, I 81:527-536.

14. Sette A, Buus S, Appella E, Smith JA, Chesnut R, Miles C, Colon SM, Grey HM: Prediction of major histocompatibility complex binding regions of protein antigens by sequence pattern analysis. Proc Natl Acad Sci USA 1989, 86:3296-3300.

15. Rotzschke O, Falk K, Stevanovic S, Jung G, Walden P, Rammensee HG: Exact prediction of a natural $\mathbf{T}$ cell epitope. Eur J Immunol |99|, 2 |:289|-2894.

16. Parker KC, Bednarek MA, Coligan JE: Scheme for ranking potential HLA-A2 binding peptides based on independent binding of individual peptide side-chains. J Immunol I 994, I 52: I 63-I75.

17. Hammer J, Bono E, Gallazzi F, Belunis C, Nagy Z, Sinigaglia F: Precise prediction of major histocompatibility complex class II-peptide interaction based on peptide side chain scanning. J Exp Med 1994, 1 80:2353-2358.

18. Southwood S, Sidney J, Kondo A, del Guercio MF, Appella E, Hoffman S, Kubo RT, Chesnut RW, Grey HM, Sette A: Several common HLA-DR types share largely overlapping peptide binding repertoires. J Immunol 1998, I 60:3363-3373.

19. Rammensee H, Bachmann J, Emmerich NP, Bachor OA, Stevanovic S: SYFPEITHI: database for MHC ligands and peptide motifs. Immunogenetics 1999, 50:213-219.

20. de Lalla C, Sturniolo T, Abbruzzese L, Hammer J, Sidoli A, Sinigaglia F, Panina-Bordignon P: Cutting edge: identification of novel $\mathbf{T}$ cell epitopes in Lol $\mathbf{p 5 a}$ by computational prediction. J Immunol 1999, I63:1725-1729.

21. Panigada M, Sturniolo T, Besozzi G, Boccieri MG, Sinigaglia F, Grassi GG, Grassi F: Identification of a promiscuous T-cell epitope in Mycobacterium tuberculosis Mce proteins. Infect Immun 2002, 70:79-85

22. Schroers R, Huang XF, Hammer J, Zhang J, Chen SY: Identification of HLA DR7-restricted epitopes from human telomerase reverse transcriptase recognized by CD4+ T-helper cells. Cancer Res 2002, 62:2600-2605.

23. Korber $B$, LaBute $M$, Yusim $K$ : Immunoinformatics comes of age. PLoS Comput Biol 2006, 2:e7I.

24. Tsurui H, Takahashi T: Prediction of T-cell epitope. J Pharmacol Sci 2007, I 05:299-316.

25. Bartnes K, Leon F, Briand JP, Travers PJ, Hannestad K: N-terminal elongation of a peptide determinant beyond the first primary anchor improves binding to H-2 I-Ad and HLA-DRI by backbone-dependent and aromatic side chain-dependent interactions, respectively. Eur J Immunol I999, 29: I89-195.

26. Shulman NR, Aster RH, Leitner A, Hiller MC: Immunoreactions involving platelets. V. Post-transfusion purpura due to a complement-fixing antibody against a genetically controlled platelet antigen. A proposed mechanism for thrombocytopenia and its relevance in "autoimmunity.". J Clin Invest 196I, 40:1597-1620.

27. Newman PJ, Derbes RS, Aster RH: The human platelet alloantigens, PIAI and PIA2, are associated with a leucine33/ proline 33 amino acid polymorphism in membrane glycoprotein IIIa, and are distinguishable by DNA typing. J Clin Invest 1989, 83: I778-I78|.

28. Parry CS, Gorski J, Stern LJ: Crystallographic structure of the human leukocyte antigen DRA, DRB3*0IOI: models of a directional alloimmune response and autoimmunity. J Mol Biol 2007, 37 I:435-446.

29. Yassai M, Afsari A, Garlie J, Gorski J: C-terminal anchoring of a peptide to class II MHC via the PIO residue is compatible with a peptide bulge. J Immunol 2002, I 68: I28I-I 285.

30. O'Sullivan D, Sidney J, Del Guercio MF, Colon SM, Sette A: Truncation analysis of several DR binding epitopes. J Immunol I99I, | 46: | 240-1246.

31. Zavala-Ruiz Z, Strug I, Anderson MW, Gorski J, Stern LJ: A polymorphic pocket at the $P I 0$ position contributes to peptide bind- 
ing specificity in class II MHC proteins. Chem Biol 2004, I I:I395-1402.

32. Geluk A, van Meijgaarden KE, Schloot NC, Drijfhout JW, Ottenhoff TH, Roep BO: HLA-DR binding analysis of peptides from islet antigens in IDDM. Diabetes 1998, 47:1594-1601.

33. Sturniolo T, Bono E, Ding J, Raddrizzani L, Tuereci O, Sahin U, et al.: Generation of tissue-specific and promiscuous HLA ligand databases using DNA microarrays and virtual HLA class II matrices. Nat Biotechnol 1999, 17:555-56I.

34. Bui HH, Sidney J, Peters B, Sathiamurthy M, Sinichi A, Purton KA, Mothe BR, Chisari FV, Watkins DI, Sette A: Automated generation and evaluation of specific MHC binding predictive tools: ARB matrix applications. Immunogenetics 2005, 57:304-3I4.

35. MacArthur MW, Thornton JM: Influence of proline residues on protein conformation. J Mol Biol I99I, 21 8:397-4I2.

36. McFarland BJ, Katz JF, Sant AJ, Beeson C: Energetics and cooperativity of the hydrogen bonding and anchor interactions that bind peptides to MHC class II protein. J Mol Biol 2005, 350: $170-183$.

37. McFarland BJ, Beeson C, Sant AJ: Cutting edge: a single, essential hydrogen bond controls the stability of peptide-MHC class II complexes. J Immunol 1999, 163:3567-357I.

38. Sant AJ, Beeson C, McFarland B, Cao J, Ceman S, Bryant PW, Wu S: Individual hydrogen bonds play a critical role in MHC class II: peptide interactions: implications for the dynamic aspects of class II trafficking and DM-mediated peptide exchange. Immunol Rev 1999, I 72:239-253.

39. Pashine A, Busch R, Belmares MP, Munning JN, Doebele RC, Buckingham M, Nolan GP, Mellins ED: Interaction of HLA-DR with an acidic face of HLA-DM disrupts sequence-dependent interactions with peptides. Immunity 2003, 19:183-192.

40. Peters B, Tong W, Sidney J, Sette A, Weng Z: Examining the independent binding assumption for binding of peptide epitopes to MHC-I molecules. Bioinformatics 2003, 19:1765-1772.

4I. Li Y, Li H, Martin R, Mariuzza RA: Structural basis for the binding of an immunodominant peptide from myelin basic protein in different registers by two HLA-DR2 proteins. J Mol Biol 2000, 304: 177-I88.

42. Scott CA, Peterson PA, Teyton L, Wilson IA: Crystal structures of two I-Ad-peptide complexes reveal that high affinity can be achieved without large anchor residues. Immunity 1998, 8:319-329.

Publish with Bio Med Central and every scientist can read your work free of charge

"BioMed Central will be the most significant development for disseminating the results of biomedical research in our lifetime. "

Sir Paul Nurse, Cancer Research UK

Your research papers will be:

- available free of charge to the entire biomedical community

- peer reviewed and published immediately upon acceptance

- cited in PubMed and archived on PubMed Central

- yours - you keep the copyright
BioMedcentral 